\title{
Impurity Scattering of Wave Packets on a Lattice
}

\author{
Wonkee Kim ${ }^{1}$, L. Covaci $^{1}$, and F. Marsiglio ${ }^{1-3}$ \\ ${ }^{1}$ Department of Physics, University of Alberta, Edmonton, Alberta, Canada, T6G 2J1 \\ ${ }^{2}$ DPMC, Université de Genève, 24 Quai Ernest-Ansermet, CH-1211 Genève 4, Switzerland, \\ ${ }^{3}$ National Institute for Nanotechnology, National Research Council of Canada, Edmonton, Alberta, Canada, T6G 2V4
}

(Dated: August 16, 2018)

\begin{abstract}
Quantum transport in a lattice is distinct from its counterpart in continuum media. Even a free wave packet travels differently in a lattice than in the continuum. We describe quantum scattering in a one dimensional lattice using three different formulations and illustrate characteristics of quantum transport such as resonant transmission. We demonstrate the real time propagation of a wave packet and its phase shift due to impurity configurations. Spin-flip scattering is also taken into account in a spin chain system. We show how individual spins in the chain evolve as a result of a spin-flip interaction between an incoming electron and a spin chain.
\end{abstract}

PACS numbers: 72.10.-d, 73.50.Bk, 73.21.Hb

\section{INTRODUCTION}

In the last few decades the advent of pump-probe optical methods $\frac{1,2.3}{}$ and similar measurement techniques has stimulated interest in time-dependent phenomena in physical systems $\stackrel{4}{4}$ For example, questions concerning the details of magnetization reversal in ferromagnetic thin films can be addressed experimentally ${ }^{\underline{5}}$ and theoretically ${ }^{6}$. Another example is polaron formation in a semiconductor, where both experimental and theoretical work are just starting $\underline{\underline{7}}$ While much of the work on magnetization reversal has utilized a classical or at most semi-classical description,,$\frac{8,9.10,11,12.13}{13}$ more recent work has focused on a fully quantum mechanical description 14 Such a microscopic description is expected to be necessary and insightful for small (i.e. quantum dot) systems.

Insofar as many of these phenomena occur in the solid state, the underlying lattice structure may play an important role. Concerning the theoretical description of magnetization reversal, calculations to date have either focused on simple models $\frac{8.9 .10 .11 .15}{{ }^{2}}$ or have tried to utilize realistic transport equations with band structures relevant to the materials of interest 16 In this work we wish to solve simple scattering models based on tightbinding band structures. While we will consider mainly scalar potential scattering, the formalism is extendable to spin-flip scattering, which will be discussed. In particular, an incoming electron can scatter off a ferromagnetic thin film modelled by a Heisenberg Hamiltonian, and we can monitor the real time reaction of the magnetization to the onslaught of electrons with a completely quantum mechanical description. We also want to utilize a framework that is amenable to numerical calculation. By this we mean the following: as interactions are introduced, problems will become formidable by analytical means and large scale computation will be required. Most often this means Monte Carlo methods (at least this is so in equilibrium and linear response calculations so far) which are often well-suited to simple lattices. For these reasons we believe it is beneficial to have a latticebased framework to tackle non-equilibrium phenomena in solid state systems.

We begin with a description of the non-interacting electron, where already a novel property emerges due to the lattice: the degree of spreading of a propagating wave packet can be controlled by judicious choice of the electron energy. This is always true in one dimension and has more limited validity in higher dimensions. Next we solve the scattering problem for a rectangular potential barrier and other, simple barriers (or wells) that enter into impurity problems. We outline the methodology to solve the problem numerically, and use some illustrative examples to demonstrate the accuracy and efficiency of these calculations. Finally, we discuss spin flip scattering in a spin chain.

This paper is organized as follows. In Section. II, we describe wave packet transport in a lattice without scattering, and a possible solitonic behavior which is impossible in the continuum limit. In order to illustrate quantum scattering off impurities in a lattice, we use a quantum mechanical approach and the transfer matrix formalism, and compare the two methods using an example in Section III. A direct diagonalization of the Hamiltonian will illustrate the time evolution of a wave packet in a lattice with embedded impurities in Section IV. In Section V, we briefly explain a procedure to study spin flip scattering on a lattice. In section VI we summarize our results and outline possible future directions.

\section{WAVE PACKET TRANSPORT}

In order to understand the differences between calculations of quantum mechanical phenomena in a lattice from their counterpart in the continuum limit, it is appropriate to begin with transport of a free wave packet.

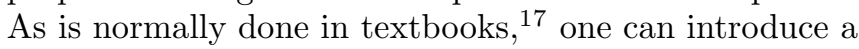
free Gaussian wave packet in the continuum limit:

$$
\Psi(x, 0)=\frac{1}{\left(2 \pi \alpha^{2}\right)^{1 / 4}} e^{-\frac{1}{4}\left(x-x_{0}\right)^{2} / \alpha^{2}+i k_{0}\left(x-x_{0}\right)}
$$


where $x_{0}$ and $k_{0}$ are the mean position and momentum, respectively, of the wave packet, and $\alpha$ is the position uncertainty associated with the wave packet. To see the time evolution of the wave packet we expand $\Psi(x, 0)$ in terms of the momentum eigenstates $|k\rangle$. They are the known solutions to the Schrodinger equation in free space; hence the time dependent Schrodinger equation is readily solved in this basis. The result is 17

$$
\Psi(x, t)=\left(\frac{\alpha^{2}}{2 \pi}\right)^{1 / 4} \frac{e^{i\left(k_{0} x-E_{0} t\right)}}{\sqrt{\alpha^{2}+i t / 2 m}} e^{-\frac{1}{4}\left(x-v_{0} t\right)^{2} /\left(\alpha^{2}+i t / 2 m\right)}
$$

where $E_{0}=k_{0}^{2} / 2 m$ and $v_{0}=k_{0} / m$ are the average energy and particle velocity, respectively. Since $\langle x\rangle=v_{0} t$ and $\left\langle x^{2}\right\rangle=\left(v_{0} t\right)^{2}+\left[\alpha^{2}+(t / 2 m \alpha)^{2}\right]$, the position uncertainty $\Delta x$ defined as $(\Delta x)^{2}=\left\langle x^{2}\right\rangle-\langle x\rangle^{2}$ becomes $\Delta x=\sqrt{\alpha^{2}+t^{2} /(2 m \alpha)^{2}}$. Similarly, we obtain the momentum uncertainty $\Delta k=1 /(2 \alpha)$. The uncertainty relation is therefore

$$
\Delta x \cdot \Delta k=\frac{1}{2} \sqrt{1+\left(\frac{t}{2 m \alpha^{2}}\right)^{2}} .
$$

This means that the uncertainty relation increases as a function of time. Also note that the relation does not depend on the mean momentum of the wave packet; as we will see, this is true only for a parabolic energy dispersion.

In a one dimensional lattice described by a nearest neighbor tight-binding model, $E_{k}=-2 t_{0} \cos (k a)$, where $t_{0}$ is a hopping amplitude to the nearest neighbor site and $a$ is a lattice constant. Hereafter we set $a=1$ and use it as the unit of length. The position is now discrete and represented by $x_{i}$, with $i$ the lattice site label. Upon expanding in terms of the momentum eigenstates in a box with periodic boundary conditions, one obtains

$$
\Psi\left(x_{i}, t\right)=\left(\frac{\alpha^{2}}{2 \pi^{3}}\right)^{1 / 4} \int_{-\pi}^{\pi} d k e^{i k x_{i}-\alpha^{2}\left(k-k_{0}\right)^{2}-i E_{k} t} .
$$

Note that the integration is over a Brillouin zone, due to the discreteness of the lattice; nonetheless, if $\alpha$ is large enough, the integration range can be extended from $-\infty$ to $\infty$ without altering the integral. In the same way, using a large $\alpha$ expansion for the exponent in Eq. (4), and keeping terms up to $\mathcal{O}\left(1 / \alpha^{2}\right)$, one can convert the integral into a Gaussian integral, as in the continuum limit. Performing the integration, we obtain

$$
\Psi\left(x_{i}, t\right)=\left(\frac{\alpha^{2}}{2 \pi^{3}}\right)^{1 / 4} \frac{\sqrt{\pi}}{\sqrt{\alpha^{2}+i t E_{k_{0}}^{\prime \prime} / 2}} e^{i k_{0} x_{i}-i E_{k_{0}} t} e^{-\frac{1}{4}\left[x_{i}-t E_{k_{0}}^{\prime}\right]^{2} /\left[\alpha^{2}+i t E_{k_{0}}^{\prime \prime} / 2\right]},
$$

where we restored more generality (than in Eq. (4) by using $E_{k_{0}}^{\prime}$ and $E_{k_{0}}^{\prime \prime}$ to refer to the first and second derivatives of the dispersion, $E_{k}$, with respect to momentum $k$, and evaluated at $k_{0}$. For a quadratic dispersion one readily obtains the result Eq. 2 On the other hand, for the nearest neighbor model, $E_{k_{0}}^{\prime} \equiv v_{k_{0}}=2 t_{0} \sin \left(k_{0}\right)$ and $E_{k_{0}}^{\prime \prime} \equiv v_{k_{0}}^{\prime}=2 t_{0} \cos \left(k_{0}\right)$, where $v_{k_{0}}$ is the group velocity, and $v_{k_{0}}^{\prime}$ is the group velocity dispersion. The expansion is valid as long as $t \ll\left(\alpha / k_{0}\right)^{3} / t_{0}$. For $k_{0}=\pi / 2$, the validity length $l$ is $l=v_{k_{0}} t \sim \alpha^{3}$. If $\alpha$ is on a nanometer scale, $l$ is of order $1 \mu \mathrm{m}$.

We are now able to calculate the uncertainty relation for the lattice case with nearest neighbor hopping only, at any time $t:\langle x\rangle=2 t_{0} \sin \left(k_{0}\right) t$ and $\left\langle x^{2}\right\rangle=\left(2 t_{0} \sin \left(k_{0}\right) t\right)^{2}+$ $\alpha^{2}+\left(t_{0} \cos \left(k_{0}\right) t / \alpha\right)^{2}$. The uncertainty in position is then $\Delta x=\sqrt{\alpha^{2}+t_{0}^{2} \cos ^{2}\left(k_{0}\right) t^{2} / \alpha^{2}}$. The uncertainty in the momentum is the same as in the continuum limit; namely, $\Delta k=1 / 2 \alpha$. Consequently, the uncertainty relation for this case on a lattice is

$$
\Delta x \cdot \Delta k=\frac{1}{2} \sqrt{1+\frac{t_{0}^{2} \cos ^{2}\left(k_{0}\right) t^{2}}{\alpha^{4}}} .
$$

As one can see from this expression, in general the uncertainty never decreases as a function of time; the degree of increase depends on the mean momentum. However, if $k_{0}=\pi / 2$, the uncertainty remains unchanged. In other words, the wave packet possesses a solitonic behavior without showing the seemingly inevitable quantum spreading. We will demonstrate this fact numerically later. This possibility is actually well known in optics, where one seeks a medium with zero group velocity dispersion to minimize loss $\frac{18}{18}$ Nevertheless, this appears to be less appreciated in quantum mechanics.

This result persists in one dimension for any dispersion. That is, one can show that some wave vector always exists for which the group velocity dispersion is zero. In higher dimensions the situation is not quite as simple. The result remains for nearest neighbor hopping only. For example in three dimensions we have $E_{k}=-2 t_{0}\left[\cos \left(k_{x} a\right)+\cos \left(k_{y} a\right)+\cos \left(k_{z} a\right)\right]$, and one readily obtains a similar result as in one dimension. However, when next nearest neighbor hopping is included, a little algebra shows that in general one cannot achieve conditions for zero group velocity dispersion.

To obtain this result numerically, one diag- 
Fig. 1 (Kim et al)

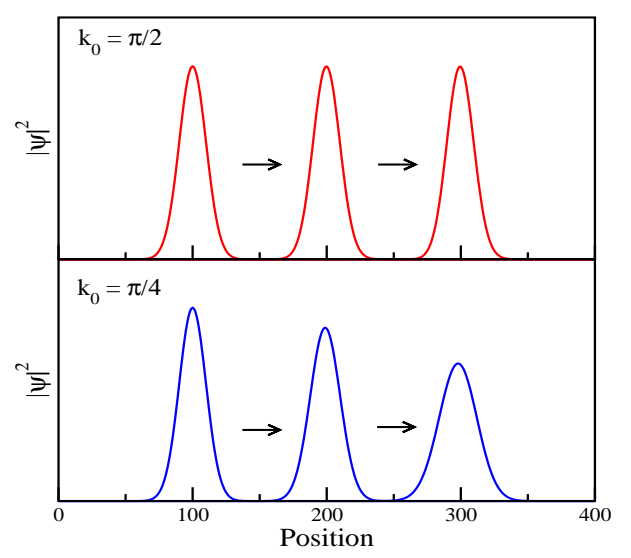

FIG. 1: Time evolution of the wave packet with $k_{0}=\pi / 2$ (top) and $\pi / 4$ (bottom). The wave packet with $k_{0}=\pi / 2$ does not spread while the wave packet $k_{0}=\pi / 2$ broadens as it moves.

onalizes the tight-binding Hamiltonian, $H_{0}=$ $-t_{0} \sum_{i}\left[C_{i}^{+} C_{i+1}+C_{i+1}^{+} C_{i}\right]$ to obtain eigenvalues and the corresponding eigenstates. Then one can construct a wave packet, and evolve it in time according to the time dependent Schrodinger equation (this procedure is described in more detail in Section IV). The result is plotted in Fig. 1 for three different times with $k_{0}=\pi / 2$ $\left(k_{0}=\pi / 4\right)$ in the top (bottom) panel, respectively. A wave packet initially centered at $x_{i}=100$ moves to $x_{i} \simeq 300$ in each panel. The uncertainty parameter $\alpha$ is set to be 10. The total time elapsed is different in the two panels because of the $k_{0}$ difference (in a consistent dimensionless time unit, 100 for the top, and 140 for the bottom panel). As one can see, the wave packet with $k_{0}=\pi / 2$ does not spread and the peak height remains unchanged while the wave packet with $k_{0}=\pi / 4$ spreads and the height becomes considerably smaller as it moves.

\section{QUANTUM MECHANICAL APPROACH}

In the previous section we examined the free wave packet transfer in a lattice. While construction of a packet proceeds as in the continuum case, the tightbinding dispersion leads to a variety of possible wave packet characteristics. In this section we examine the consequences for scattering, in one dimension. The electronic transfer can be explored based on the quantum mechanical approach using the matching conditions of wave functions, or through the transfer matrix formalism, or through direct diagonalization of the Hamiltonian. The quantum mechanical approach and the transfer matrix formalism are formally equivalent to each other and relatively analytical compared with direct diagonalization, which is inherently numerical and the subject of a later section. If there are $N$ impurities embedded in a lattice, one needs to deal with a $(2 N \times 2 N)$ matrix to determine all the relevant coefficients including $R$ and $T$ in the quantum mechanical approach. On the other hand, the transfer matrix formalism requires manipulation of $N(2 \times 2)$ matrices. Thus, when the number of impurities is large, say greater than 5 , the quantum mechanical approach is not as feasible as the transfer matrix formalism.

We used the transfer matrix formalism to study symmetries of electron-impurity scattering previously 21 In this paper, we will mainly use the quantum mechanical approach in a lattice and compare the two methods using several examples. Recall the situation in the continuum limit: consider a Hamiltonian $\mathcal{H}=-\partial_{x}^{2} /(2 m)+V(x)$ where, for this illustration we use a simple rectangular barrier potential: $V(x)=V_{0} \Theta(d / 2-|x|)$. In order to solve the Schrödinger equation, $\mathcal{H} \psi(x)=E_{k} \psi(x)$ with $E_{k}=k^{2} /(2 m)$, we write down the most general wave function in the three regions,

$$
\psi(x)= \begin{cases}\psi_{L}(x)=e^{i k x}+R e^{-i k x} & \text { for } x \leq-d / 2 \\ \psi_{i n}(x)=A e^{i q x}+B e^{-i q x} & \text { for }|x| \leq d / 2 \\ \psi_{R}(x)=T e^{i k x} & \text { for } x \geq d / 2\end{cases}
$$

where $q$ is determined by $q=\sqrt{2 m\left(E_{k}-V_{0}\right)}$. Note that $\psi_{L, R}(x)$ describes a free electron satisfying $\mathcal{H}_{0} \psi_{L, R}(x)=$ $E \psi_{L, R}(x)$ for any value of $x$, where $\mathcal{H}_{0}=-\partial_{x}^{2} /(2 m)$. Using the matching conditions of $\psi(x)$ and $\partial \psi(x) / \partial x$ at $x= \pm d / 2$, we determine the coefficients $R, A, B$, and $T$. An example $\frac{19}{9}$ of this case is the one as $d \rightarrow 0$ and $V_{0} \rightarrow \infty$ while $d V_{0}$ is finite; namely, $V(x)=d V_{0} \delta(x)$. A little algebra yields $|T|^{2}=1 /\left[1+\left(d V_{0} / v_{k}\right)^{2}\right]$ and $|R|^{2}=$ $1-|T|^{2}$, where the velocity $v_{k}=\partial E_{k} / \partial k=k / m$.

In a lattice, a potential is represented by a series of "impurities" whose effect is to alter the on-site energy wherever an impurity has substituted for the usual atom. This is represented by the Hamiltonian,

$$
H=-t_{0} \sum_{i}\left[C_{i}^{+} C_{i+1}+C_{i+1}^{+} C_{i}\right]+\sum_{i \in \mathcal{I}} U_{i} C_{i}^{+} C_{i},
$$

where $t_{0}$ is the hopping amplitude as before, $C_{i}^{+}$creates an electron at a site $i$, and $U_{i}$ is a scalar potential at site $i$; the set of "impurities" spans a number $\mathcal{I}=\{0,1,2, \cdots, I\}$. Note that $U_{i}$ can be positive (repulsive), negative (attractive), or zero. In this way one can construct any shape potential one wishes (on a scale much greater than the lattice spacing). Since we consider only scalar potentials, and we are treating the one electron problem here, we ignore the spin index of the electrons. The wave function defined only on the lattice sites can now be written as a piecewise function over $I+3$ regions since the number of impurities is $I+1$. That is, $|\psi\rangle=\sum_{j} \psi\left(x_{j}\right) C_{j}^{+}|0\rangle$, where

$$
\psi\left(x_{j}\right)= \begin{cases}\psi_{L}\left(x_{j}\right)=e^{i k x_{j}}+R e^{-i k x_{j}} & \text { for } j<0 \\ \psi_{j}\left(x_{j}\right)=A_{j} e^{i q_{j} x_{j}}+B_{j} e^{-i q_{j} x_{j}} & \text { for } j \in \mathcal{I} \\ \psi_{R}\left(x_{j}\right)=T e^{i k x_{j}} & \text { for } j>I\end{cases}
$$


and $|0\rangle$ represents the vacuum, namely, the state with an empty lattice. Since we set the lattice constant to be unity, the displacement $x_{i}=a \cdot i=i$. The coefficients $R, A_{i}, B_{i}$, and $T$ are to be determined by matching conditions at $i \in \mathcal{I}$. Note that $q_{i}$ will be obtained within the same calculation even though we can already guess that $E_{k}=E_{q_{i}}+U_{i}$. What are the matching conditions ? Like the continuum limit one first demands continuity of the wave function at each site. Therefore, $\left\langle 0\left|C_{j+}\right| \psi\right\rangle=\left\langle 0\left|C_{j-}\right| \psi\right\rangle$, where $j+(j-)$ means just to the right (left) of site $j$. However, the second condition in the continuum limit requires continuity of the derivative of the wave functions at each site. One can see this directly from the Schrödinger equation through an integration of the second order differential equation. However, the second quantized form of the Hamiltonian written in Eq. (7) contains no derivatives, so clearly this procedure is not an option. The correct procedure is as follows 20 One first writes down the Schrödinger equation projected onto each site, $\left\langle 0\left|C_{j} H\right| \psi\right\rangle=\left\langle 0\left|C_{j} E\right| \psi\right\rangle$ for $j=0,1, \cdots, I$. Then the two conditions, expressed for each site, can be written

$$
\psi\left(j+0^{+}\right)=\psi\left(j+0^{-}\right)
$$

$$
-t_{0}[\psi(j+1)+\psi(j-1)]+U_{j} \psi(j)=E \psi(j) .
$$

As mentioned before, Eq. (8) implies the wave function is continuous at each site. Eq. (9) is the Schrödinger equation at each site; however, close inspection shows that on the left-hand side the wave function is required from two different "pieces" in the domain (assuming that $U_{j}$ is non-zero. But we would like the Schrödinger equation for non-interacting electrons to be satisfied, with the same eigenvalue, by the wave function on any given "piece" even when extended beyond the domain of validity of that wave function. For example, for $j=0$ we demand that $\psi_{L}$ satisfy the equation, $-t_{0}\left[\psi_{L}(+1)+\psi_{L}(-1)\right]=$ $E \psi_{L}(0)$. Note that we have used the wave function $\psi_{L}$ at location +1 , even though it was originally defined only for sites 0 or below. Moreover, we require that this equation be satisfied with the same eigenvalue, $E$. Hence, by judicious adding and subtracting of a wave function to Eq. (9) at each impurity site, we arrive at, for $j=0$ and $j=I$ :

$$
\begin{aligned}
-t_{0} \psi_{1}(1)+t_{0} \psi_{L}(1)+U_{0} \psi_{L}(0) & =0 \text { for } j=0 \\
-t_{0} \psi_{I}(I-1)+t_{0} \psi_{R}(I-1)+U_{I} \psi_{R}(I) & =0 \text { for } j=I .
\end{aligned}
$$

Similar equations apply for the impurity sites in between. These can now be solved for the unknown coefficients along with the "matching equations" [Eq. (8)].

Let us consider a two impurity case as an example. Assume two impurities with $U_{0}$ and $U_{1}$ are embedded at $j=0$ and 1 , respectively, in a lattice; one needs to introduce a wave function as follows:

$$
\psi\left(x_{j}\right)=\left\{\begin{array}{l}
\psi_{L}\left(x_{j}\right)=e^{i k x_{j}}+R e^{-i k x_{j}} \\
\psi_{0}\left(x_{j}\right)=A_{0} e^{i q_{0} x_{j}}+B_{0} e^{-i q_{0} x_{j}} \\
\psi_{1}\left(x_{j}\right)=A_{1} e^{i q_{1} x_{j}}+B_{1} e^{-i q_{1} x_{j}} \\
\psi_{R}\left(x_{j}\right)=T e^{i k x_{j}}
\end{array}\right.
$$

The coefficients such as $R$ and $T$, and the momenta $q_{0}$, and $q_{1}$ can be determined by solving the continuity equations $\psi_{L}(-1)=\psi_{1}(-1), \psi_{L}(0)=\psi_{1}(0), \psi_{1}(0)=$ $\psi_{2}(0), \psi_{1}(1)=\psi_{2}(1), \psi_{2}(1)=\psi_{R}(1), \psi_{1}(2)=\psi_{R}(2)$, and the Schrödinger equations

$$
\begin{aligned}
& -\psi_{1}(1)+\psi_{L}(1)+U_{0} \psi_{L}(0)=0 \\
& -\psi_{1}(0)+\psi_{R}(0)+U_{1} \psi_{R}(1)=0
\end{aligned}
$$

where we set the nearest neighbor hopping amplitude $t_{0}$ to be unity for simplicity. It is straightforward to show that

$$
T=\frac{2 i \sin (k)}{2 i \sin (k)-\left(U_{0}+U_{1}\right)-U_{0} U_{1} e^{i k}}
$$

$$
R=\frac{U_{0}+U_{1} e^{2 i k}+U_{0} U_{1} e^{i k}}{2 i \sin (k)-\left(U_{0}+U_{1}\right)-U_{0} U_{1} e^{i k}} .
$$

Using the Schrödinger equations, one can show that $E_{k}=E_{q_{i}}+U_{i}(i=1,2)$. This result will be used to compare the quantum mechanical approach and the transfer matrix formalism. A case of two impurities with same potentials $\left(U_{0}=U_{1}\right)$ has been well studied in a context of the random dimer model $22,23.24$

In Fig. 2, we plot $|T|^{2}$ for two impurities side by side using Eq. (13). Fig. 2(a) to Fig. 2(c) are contour plots of $\left|T\left(E, U_{1}\right)\right|^{2}$ for given values of $U_{0}=-1,1$, and 1.8, respectively. Note that Fig. 2(a) and Fig. 2(b) illustrate a symmetry, $\left|T\left(E, U_{0}, U_{1}\right)\right|^{2}=\left|T\left(-E,-U_{0},-U_{1}\right)\right|^{2}$, which we showed in a previous paper ${ }^{21}$ When we increase $U_{0}$ further, $|T|^{2}$ becomes suppressed considerably as shown in Fig. 2(c). We also plot $|T|^{2}$ vs. $E$ in Fig. 2(d) for various values of $U_{1}$ while $U_{0}$ is fixed to be 1.0. In fact, Fig. 2(d) can be read off from Fig. 2(b) along a line of the corresponding value of $U_{1}$. In the case of two impurities, a transmission resonance $\left(|T|^{2}=1\right)$ occurs when $E=U_{0}=U_{1}$ and $|E| \leq 2$. As one can see in Fig. 2(d), a transmission resonance $\left(|T|^{2}=1\right)$ occurs at $E=1$ for $U_{0}=U_{1}=1$. 

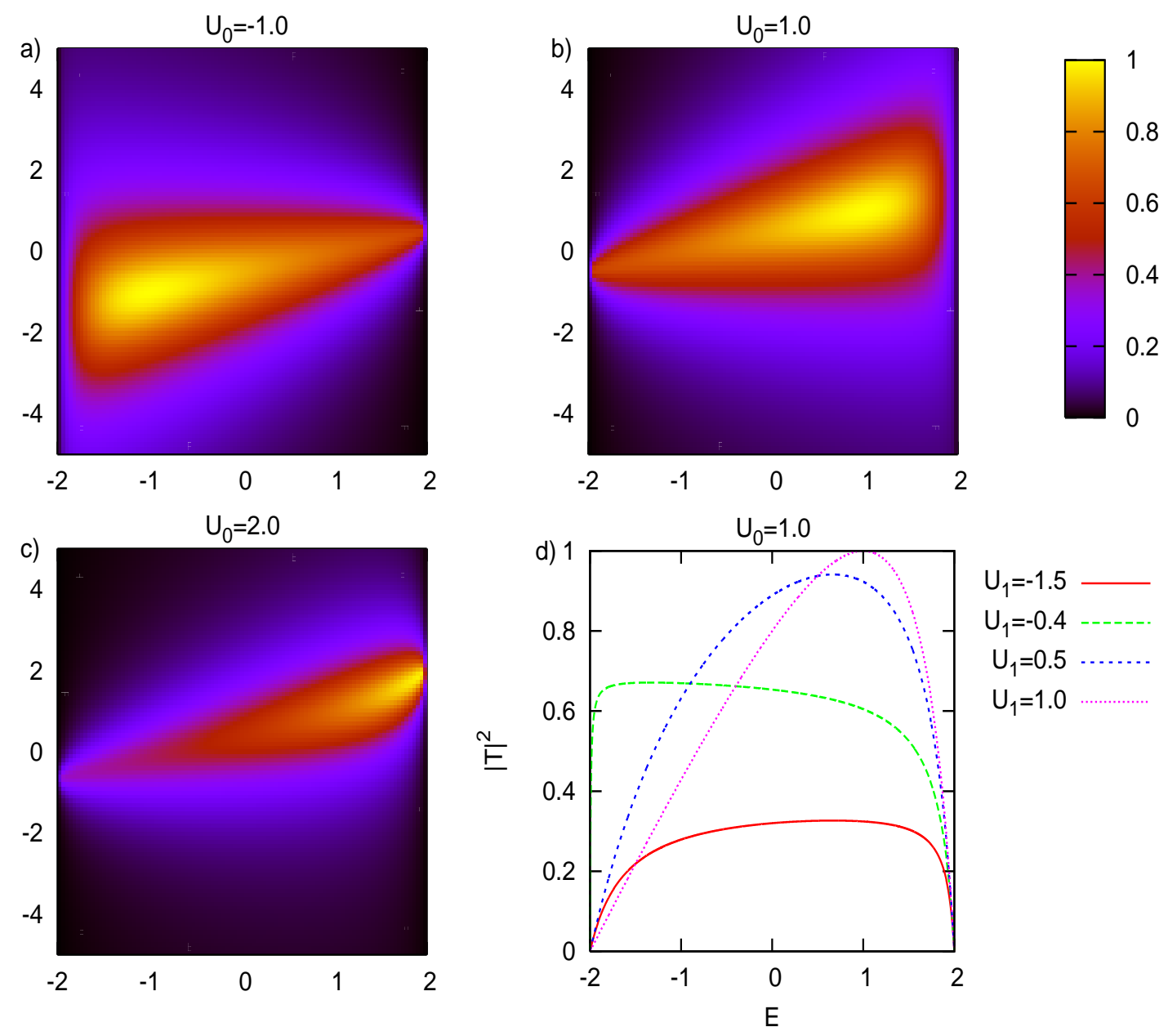

FIG. 2: $|T|^{2}$ for two impurities using Eq. (13). Fig. 2(a) to Fig. 2(c) are contour plots of $\left.T\left(E, U_{1}\right)\right|^{2}$ for given values of $U_{0}=-1,1$, and 1.8, respectively. Fig. $2(\mathrm{~d})$ is $|T|^{2}$ as a function of $E$ for various values of $U_{1}$ with $U_{0}=1.0$.

For three impurities with $U_{0}, U_{1}$, and $U_{2}$ at $j=0,1$ mission amplitude and 2 , respectively, a similar calculation gives the trans-

$$
T=\frac{-2 i \sin (k)}{\left(U_{1}-E_{k}\right)+e^{i k}\left[\left(U_{0}+U_{2}\right)\left(U_{1}-E_{k}\right)-2\right]+e^{2 i k}\left[U_{0} U_{2}\left(U_{1}-E_{k}\right)-\left(U_{0}+U_{2}\right)\right]} .
$$

It is easy to show that Eq. (15) reduces to Eq. (13) as $U_{0}$ or $U_{2}$ goes to zero. Giri et al ${ }^{25}$ studied this case in detail and classified the parameter values into five cases, for which perfect transmission can be obtained. The first is the dimer case, and the second only applies if the hopping parameters in the impurity region are different, so here we will consider only the remaining three cases. Let us briefly describe the three cases using our notation. In case (III), $U_{0}=U_{1}=U_{2}$ and $E=U_{0} \pm 1$. This is the straightforward extension of the symmetric dimer case to a symmetric trimer, but note that now the potential for two solutions exists. In case (IV), if $U_{0}+U_{2}=U_{1}$ then resonance is obtained at an energy, $E=U_{1}$, regardless of the values of $U_{0}$ and $U_{2}$. We will note an example 

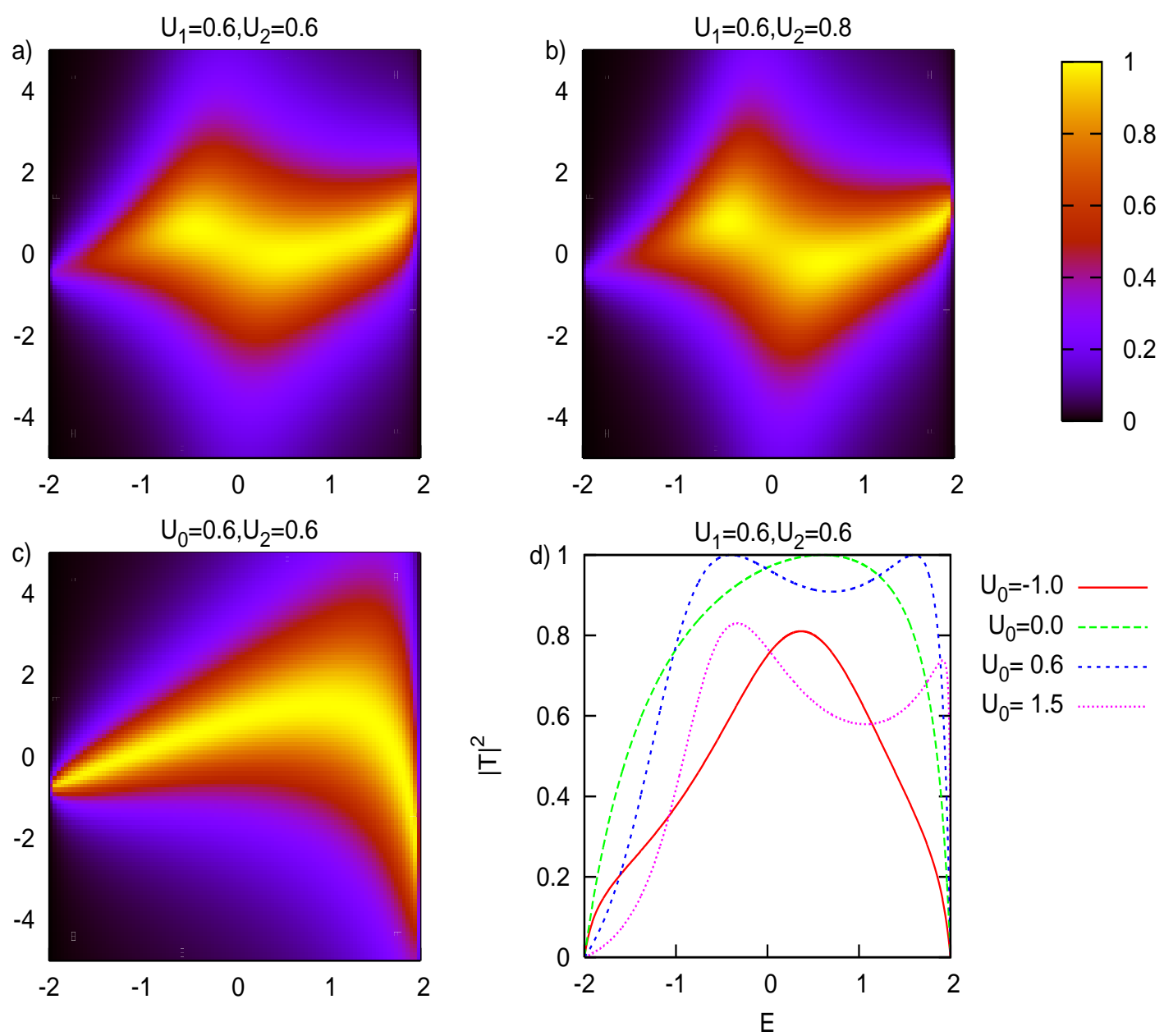

FIG. 3: $|T|^{2}$ for three impurities using Eq. (15). Fig. 3(a) to Fig. 3(c) are contour plots while Fig. $3\left(\right.$ d) is for $|T|^{2}$ vs. $E$. Fig. 3(a) is for $\left|T\left(E, U_{0}\right)\right|^{2}$ with $U_{1}=U_{2}=0.6$. Fig. 3(b) is also for $\left|T\left(E, U_{0}\right)\right|^{2}$ but with $U_{1}=0.6$ and $U_{2}=0.8$. Fig. 3(c) shows $\left|T\left(E, U_{1}\right)\right|^{2}$ with $U_{0}=U_{2}=0.6$. Fig. $3(\mathrm{~d})$ is for $|T|^{2}$ vs. $E$, which can be read off from Fig. 3(a) along a line of the corresponding value of $U_{0}$.

below. Case (V) is the most interesting; in this instance $U_{0}=U_{2}$ and $U_{1}$ is arbitrary. Then resonance is obtained for energies,

$$
E=U_{1}+\frac{1}{2}\left[U_{1}-U_{0} \pm \sqrt{\left(U_{1}-U_{0}\right)^{2}-4\left(U_{1} / U_{0}-2\right)}\right]
$$

provided that $\left(U_{1}-U_{0}\right)^{2} \geq 4\left(U_{1} / U_{0}-2\right)$ and of course $-2 \leq E \leq 2$. We plot $|T|^{2}$ for three impurities sitting side by side in Fig. 3. Fig. 3(a) to Fig. 3(c) are contour plots while Fig. 3(d) is for $|T|^{2}$ vs. E. Fig. 3(a) is for $\left|T\left(E, U_{0}\right)\right|^{2}$ with $U_{1}=U_{2}=0.6$ and illustrates both case (III) and case (IV). For example, if $U_{0}=0.6,|T|^{2}=1$ at $E=1.6$ and -0.4 . This belongs to case (III). On the other hand, since $U_{1}=U_{2}$, then when $U_{0}=0,|T|^{2}=1$ at $E=0.6$, which belongs to case (IV). In Fig. 3(b), which is also a contour plot of $\left|T\left(E, U_{0}\right)\right|^{2}$ but with $U_{1}=$ 0.6 and $U_{2}=0.8$, we still have case (IV) when $U_{0}=$ -0.2 and $E=0.6$. On the other hand, when $U_{0}=0.8$, $|T|^{2}=1$ at $E=1.82$ and -0.42 , which belongs to case
(V). Fig. 3(c) is primarily an example of case $(\mathrm{V})$ since $U_{0}=U_{2}$. The condition $\left(U_{1}-U_{0}\right)^{2} \geq 4\left(U_{1} / U_{0}-2\right)$ requires $U_{1}<1.27$ for $U_{0}=0.6$. Since $|E| \leq 2$, one finds that there exist two solutions for $|T|^{2}=1$ if $-0.75 \leq$ $U_{1} \leq 1.27$; however, for $-3<U_{1}<-0.75$ there is only one solution as can be observed in Fig. 3(c). Also note that Fig. 3(c) contains as a special case the 'spread-out' dimer, where the middle of the trimer has no impurity $\left(U_{1}=0\right)$, so the remaining dimer is now separated by two lattice spacings. This is a special example of Giri et al.'s case (V), and is also a special case of the arbitrarily spread-out dimer considered by us previously 21 .

Fig. 3(d) is for $|T|^{2}$ vs. $E$, which can be read off from Fig. 3(a) along a line of the corresponding value of $U_{0}$. Note the two solutions for perfect transmission for the symmetric trimer case (dashed blue curve, $U_{0}=0.6$ ). Also shown is a dimer case (dashed green curve), when $U_{0}=0$, for which $E=0.6$ yields resonant transmission. The standard one dimensional plots are quite clear; on the other hand the contour plots provide a feel for how 
strongly the transmission remains as parameters vary away from the resonant condition.

\section{TRANSFER MATRIX FORMALISM}

In the transfer matrix formalism, ${ }^{26}$ we write the Schrödinger equation Eq. (9) in the matrix form as follows:

$$
\left(\begin{array}{c}
\psi_{j+1} \\
\psi_{j}
\end{array}\right)=M_{j}\left(\begin{array}{c}
\psi_{j} \\
\psi_{j-1}
\end{array}\right)
$$

where $M_{j}=\left(\begin{array}{cc}U_{j}-E_{k} & -1 \\ 1 & 0\end{array}\right)$, which is a unimodular matrix and associated with an impurity at the site $j$. The wave functions $\psi_{L}($ for $i<1)$ and $\psi_{R}($ for $i>N)$ are $\psi_{L}=e^{i k x_{i}}+R e^{-i k x_{i}}$ and $\psi_{R}=T e^{i k x_{i}}$. Using the transfer matrix formalism, one can express the coefficients $R$ and $T$ in terms of $k, U_{i}$, and $E$ as follows:

$$
\left(\begin{array}{c}
\mathcal{T} \\
i \mathcal{T}
\end{array}\right)=P\left(\begin{array}{c}
1+\mathcal{R} \\
i(1-\mathcal{R})
\end{array}\right)
$$

where $P=S^{-1} M S$ with $S=\left(\begin{array}{cc}\cos (k) & \sin (k) \\ 1 & 0\end{array}\right)$, and $M=M_{N} M_{N-1} \cdots M_{1}$. Solving Eq. (18), one can $\operatorname{obtain}^{27}$

$$
\begin{aligned}
\mathcal{T} & =\frac{2 i}{i\left(P_{11}+P_{22}\right)+P_{12}-P_{21}} \\
\mathcal{R} & =\frac{P_{12}+P_{21}-i\left(P_{11}-P_{22}\right)}{i\left(P_{11}+P_{22}\right)+P_{12}-P_{21}}
\end{aligned}
$$

It is instructive to compare the quantum mechanical approach and the transfer formalism using an example. Let us consider two impurities residing side by side. In this instance we know that the transmission and reflection amplitude are Eqs. (13) and (14). In the transfer matrix formalism, one needs to calculate $P=S^{-1} M_{1} M_{0} S$ to obtain the transmission amplitude

$$
\mathcal{T}=\frac{2 i \sin (k)}{i \sin (k)\left[\left(U_{0}-E_{k}\right)\left(U_{1}-E_{k}\right)-2\right]+U_{0}+U_{1}-2 E_{k}-\cos (k)\left(U_{0}-E_{k}\right)\left(U_{1}-E_{k}\right)}
$$

Note that $T$ [Eq. (13)] is not identical with $\mathcal{T}$ [Eq. (21)]. However, while not obvious, they merely differ by a phase factor and their magnitudes are the same: $\mathcal{T}=e^{2 i k} T$. Clearly the transfer matrix method 'keeps track' of the two lattice spacings traversed as the particle is transmitted to the other side. A similar relation holds for the reflection amplitude. Consequently, the transmission (reflection) probabilities are identical; $|T|^{2}=|\mathcal{T}|^{2}$ and $|R|^{2}=|\mathcal{R}|^{2}$ in the two formalisms.

In previous work ${ }^{21}$ using the transfer matrix formalism, we derived a relation between $\mathcal{R}$ and $\mathcal{R}^{\prime}$, where $\mathcal{R}^{\prime}$ is the reflection amplitude for the reverse impurity configuration. It is

$$
\frac{\mathcal{T}^{*}\left(\mathcal{R}^{\prime}-\mathcal{R}\right)}{\mathcal{T}\left(\mathcal{R}^{\prime}-\mathcal{R}\right)^{*}}=e^{2 i k}
$$

In the example of the two impurities, $\mathcal{R}^{\prime}$ is given by $\mathcal{R}$ with $U_{0}$ and $U_{1}$ exchanged. Using Eqs. (13) and (14), one can show that $T$ and $R$ also satisfy the same relation; namely,

$$
\frac{T^{*}\left(R^{\prime}-R\right)}{T\left(R^{\prime}-R\right)^{*}}=e^{2 i k}
$$

Introducing a phase difference between $R$ and $R^{\prime}$ such as $R^{\prime}=e^{\delta} R$, we obtain

$$
e^{i \delta}=-e^{2 i k} \frac{R^{*} T}{R T^{*}} .
$$

For the case of two impurities we considered, the phase difference $\delta$ can be determined by

$$
\tan (\delta-2 k)=\frac{2 \mu \nu}{\nu^{2}-\mu^{2}}
$$

where

$$
\begin{aligned}
\mu & =U_{0}+U_{1} \cos (2 k)+U_{0} U_{1} \cos (k) \\
\nu & =U_{1} \sin (2 k)+U_{0} U_{1} \sin (k)
\end{aligned}
$$

Later, we will show that the derivative of $\delta$ with respect to $k$ can be attributed to a phase shift (or time delay) of wave functions for the reverse configuration of impurities.

\section{NUMERICAL DIAGONALIZATION AND PHASE SHIFT OF WAVE PACKETS}

We have already alluded to the numerical approach for a free wave packet transfer in section II. Since the operator $C_{i}^{+}$creates an electron at site $i$, the initial wave packet can be written as $|\Psi(0)\rangle=\sum_{i} \varphi\left(x_{i}, 0\right) C_{i}^{+}|0\rangle$, where

$$
\varphi\left(x_{i}, 0\right)=\frac{1}{\left(2 \pi a^{2}\right)^{1 / 4}} e^{i k_{0}\left(x_{i}-x_{0}\right)} e^{-\frac{1}{4}\left(x_{i}-x_{0}\right)^{2} / \alpha^{2}} .
$$

As mentioned in Sec. II, $x_{0}$ is the mean position, $k_{0}$ is the mean momentum, and $\alpha$ is the initial uncertainty associated with the position. If $\alpha$ is much larger than the 
size of the potential region, say $I$, the wave packet acts like a plane wave when it is scattered off the potential. To see the real time propagation of a wave packet in a lattice with $N_{0}$ sites in total, one needs to diagonalize the Hamiltonian Eq. (7), which is an $\left(N_{0} \times N_{0}\right)$ matrix. Since the impurity potentials are real, the Hamiltonian matrix is real and symmetric. The numerical diagonalization is done using the expert driver DSYEVX contained in the LAPACK package, which provides either selected eigenvalues and eigenvectors or the entire spectrum. Using the eigenstates $|n\rangle$ and eigenvalues $\epsilon_{n}$ obtained from the diagonalization, one can express the wave packet at time $t$ as follows:

$$
|\Psi(t)\rangle=\sum_{n=1}^{N_{0}}|n\rangle\langle n \mid \Psi(0)\rangle e^{-i \epsilon_{n} t} .
$$

The wave packet initially at $x_{0}$ moves to the potentials and scatters off impurities. In general, the wave packet is partially reflected and partially transmitted. The mathematical definitions of the reflection and transmission probabilities are $|R|^{2}=\sum_{i<0}\left|\varphi\left(x_{i}, t\right)\right|^{2}$ and $|T|^{2}=\sum_{i>I}\left|\varphi\left(x_{i}, t\right)\right|^{2}$, respectively as $t \rightarrow \infty$.

Let us consider the two impurity case again to illustrate the time evolution of a wave packet in the presence of impurities in a lattice. The impurity potentials are set to be $U_{0}=1$ and $U_{1}=3$ in units of the hopping constant $t_{0}$. We consider wave packets with average momentum that varies from $k_{0}=0.3 \pi$ to $0.9 \pi$. The time elapsed for the scattering processes to 'finish' depends on $k_{0}$; for example, for $k_{0}=0.6 \pi$ it is 160 in our dimensionless time unit, while for $k_{0}=0.8 \pi$, it would be 240 . We consider two impurity configurations, [I] and [II]. For [I], we have $\left(U_{0}, U_{1}\right)$ and for $[\mathrm{II}],\left(U_{1}, U_{0}\right)$. As we discussed earlier, the reflection amplitude will differ correspondingly; namely, $R$ for [I] while $R^{\prime}$ for [II]. Since $T=T^{\prime}$, there is no phase shift for the transmitted wave packet as shown in Ref. 21. On the other hand, the phase difference $\delta$ induces the phase shift for the reflected wave packets. This can be explained as follows: Consider a wave packet $\psi(x, 0)$ moving with $k_{0}$ to an impurity region

$$
\psi(x, 0)=\int d k g(k) e^{i k_{0}\left(x-x_{0}\right)}
$$

where $g(k) \sim e^{-\alpha\left(k-k_{0}\right)^{2}}$. After the wave packet scatters completely off the impurities at time $t_{s}$, the wave function at $t$ after $t_{s}$ would be

$$
\begin{aligned}
\psi_{[I]}(x, t) & =\int d k g_{R}(k) e^{-i k_{0}\left(x-x_{R}\right)} e^{-i E_{k} t} \\
& +\int d k g_{T}(k) e^{i k_{0}\left(x-x_{T}\right)} e^{-i E_{k} t}
\end{aligned}
$$

where an elastic scattering is assumed, $g_{R} \sim R(k) g(k)$ while $g_{T} \sim T(k) g(k)$, and $x_{R}\left(x_{T}\right)$ is the mean position of the reflected (transmitted) wave packet at $t_{s}$. For the reverse configuration [II],

$$
\psi_{[I I]}(x, t)=\int d k g_{R^{\prime}}(k) e^{-i k_{0}\left(x-x_{R}\right)} e^{-i E_{k} t}
$$

Fig.4 (Kim et al)

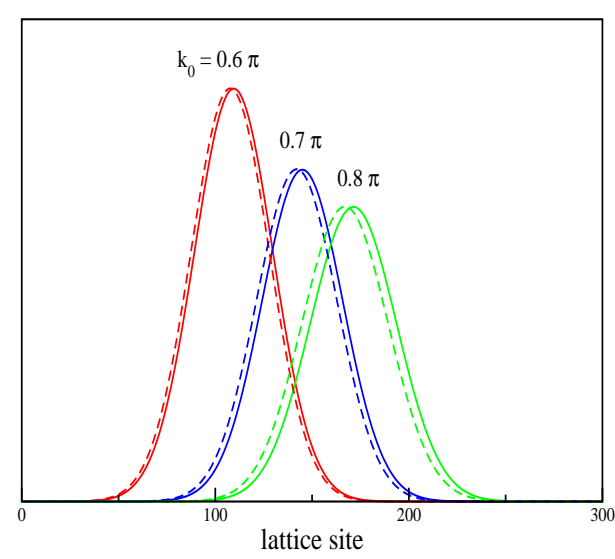

FIG. 4: Phase shift of the reflected wave packets in a lattice with two impurities embedded side by side with $U_{0}=1$ and $U_{1}=3$ in units of the hopping constant. Initially, the wave packet is at $x_{0}=150$. The two impurities are at 300 and 301 . The uncertainty parameter $\alpha$ is set to be 20 . For $\left|\psi_{[I], R}\right|^{2}$ (solid curves), the impurity configuration is $\left(U_{0}, U_{1}\right)$ while for $\left|\psi_{[I I], R}\right|^{2}$ (dashed curves), it is $\left(U_{1}, U_{0}\right)$.

$$
+\int d k g_{T^{\prime}}(k) e^{i k_{0}\left(x-x_{T}\right)} e^{-i E_{k} t}
$$

Since $R^{\prime}=R e^{i \delta}$, the reflected part of $\psi_{[I I]}(x, t)$ becomes

$$
\psi_{[I I], R}(x, t) \sim \int d k R(k) e^{-\alpha\left(k-k_{0}\right)^{2}} e^{-i k_{0}\left(x-x_{R}\right)-i E_{k} t+i \delta}
$$

In order to see the phase shift of the reflected wave packets, one needs to calculate $\left|\psi_{[I I], R}\right|^{2}$. Expanding $E(k)$ and $\delta(k)$ around $k_{0}$ and some algebra yields

$$
\left|\psi_{[I I], R}\right|^{2} \sim \exp \left[-\frac{\left(x-x_{R}+v_{0} t-\partial_{k} \delta_{0}\right)^{2}}{2\left\{\alpha^{2}+t^{2}\left(\partial_{k}^{2} E_{0}-\partial_{k}^{2} \delta_{0}\right)^{2} / 4\right\}}\right]
$$

where $v_{0}=\partial_{k} E_{0}$. Rigorously speaking, this approximation is valid when $R(k) \simeq R\left(k_{0}\right)$. Note that the term with $E_{0}$ and $\delta_{0}$ disappears when $\left|\psi_{[I I], R}\right|^{2}$ is calculated and the position of the scattered wave is $x_{R}-v_{0} t$. Eq. (34) indicates that the phase shift of the reflected wave packets is determined by $\partial_{k} \delta_{0}$, and the spreading depends not only on $\partial_{k}^{2} E_{0}$ but also on $\partial_{k}^{2} \delta_{0}$. To be specific we define a phase shift as the difference between the two reflected wave packets for [I] and [II] at their half width as in Ref. 21.

In Fig. 4, we plot the reflected wave packets for [I] (solid curves) and [II] (dashed curves) in the case of two impurities with $U_{0}=1$ and $U_{1}=3$. The average momenta for the three wave packets shown are $k_{0}=0.6 \pi$, $0.7 \pi$, and $0.8 \pi$. As one can see clearly, the phase shift between $\left|\psi_{[I], R}\right|^{2}$ (solid curves) and $\left|\psi_{[I I], R}\right|^{2}$ (dashed curves) depends on $k_{0}$. The phase shift for $k_{0}=0.6 \pi$ is not significant while it becomes bigger as $k_{0}$ increases 
Fig.5 (Kim et al)

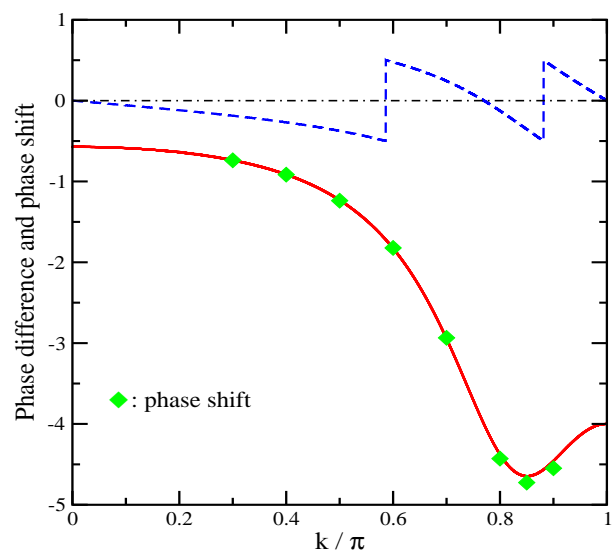

FIG. 5: Phase difference (blue dashed curve), its derivative (red solid curve), and the phase shift obtained from the wave packet simulations (green diamond symbols). The phase shift is measure by the difference between the reflected wave packets $\psi_{[I], R} \mid$ and $\psi_{[I I], R} \mid$ at their half width. The impurity potentials are $U_{0}=1$ and $U_{1}=3$. The phase shift and $\partial \delta / \partial k$ agree with one another very well.

to $0.8 \pi$. We also plot the phase difference, its derivative, and the phase shift of the reflected wave packets in Fig. 5. One may use Eq. (25) to obtain the phase difference and its derivative in this simple case. However, when we consider many impurities we need to use the transfer matrix formalism. As shown in the plot, the derivative of $\delta$ and the phase shift obtained numerically are in excellent agreement.

\section{SPIN FLIP SCATTERING}

So far we have discussed scalar potential scattering on a lattice. In this section we describe how to study spin flip scattering on a lattice. Let us consider the Hamiltonian

$H=-t_{0} \sum_{<i, j>\sigma} C_{i \sigma}^{+} C_{j \sigma}-2 J_{0} \sum_{l} \sigma_{l} \cdot \mathbf{S}_{l}-2 J_{1} \sum_{l} \mathbf{S}_{l} \cdot \mathbf{S}_{l+1}$

where $C_{i \sigma}^{+}$creates an electron with a spin $\sigma$ at a site $i, \mathbf{S}_{l}$ is a spin operator located at a site $l, t_{0}$ is a hopping amplitude between the nearest neighbor sites, and $J_{0}$ is the coupling between an electron and a local spin. The electron-spin coupling is assumed here to be a purely local (i.e. on-site) interaction. $J_{1}$ is a (Heisenberg exchange) coupling between two neighbouring spins. This model can be used to understand the spin transfer dynamics between an itinerant electron and a ferromagnetic spin chain with $N_{s}$ local spins $(S=1 / 2)$ arranged in a one dimensional lattice. To study the spin chain, one can extend the quantum mechanical approach as in Ref. 14, or one can extend the transfer matrix formalism ${ }^{28}$ Here we follow the diagonalization method to see the time evo- lution of the spins.

Suppose we send a wave packet representing an electron with spin aligned in the $+Z$ direction towards a ferromagnetic spin chain where all spins are aligned in the $-Z$ direction. Such a state of the chain will be denoted by $|G\rangle$. The incoming wave packet can be constructed as follows:

$$
|\psi(0)\rangle=\sum_{i} \varphi\left(x_{i}, 0\right) C_{i \uparrow}^{+}|0\rangle
$$

Then, the initial wave function of the total system including the incoming electron and the chain is $|\Psi(0)\rangle=$ $|\psi(0)\rangle|G\rangle$. We introduce the total spin operator $\mathbf{J}=$ $\sigma+\sum_{l} \mathbf{S}_{l}$. The $Z$ component, $J_{z}$, of the total spin is conserved. Hereafter we assume spin $1 / 2$ for both the electron and the spins, for simplicity. Since the initial value of $J_{z}$ is $\left(1-N_{s}\right) / 2$, the possible spin bases would be $|+\rangle|G\rangle$ and $|-\rangle S_{l+}|G\rangle$, where $S_{l+}$ flips the local spin at $l$ in the chain. Alternative spin bases could be used by utilizing the total spin and its $\mathrm{Z}$ component. Including the location of the incoming electron, the bases states we use are $C_{i \uparrow}^{+}|0\rangle|G\rangle$ and $C_{i \downarrow}^{+}|0\rangle S_{l+}|G\rangle$. Now, the Hamiltonian becomes an $N_{0}\left(N_{s}+1\right) \times N_{0}\left(N_{s}+1\right)$ matrix. Note that the dimension of the Hamiltonian depends only on $N$ and $N_{s}$ and does not depend on the locations of the local spins. Even if we include impurities, the dimension of the Hamiltonian remains unchanged. To construct the Hamiltonian matrix, we need to calculate each component of the matrix. For example,

$$
\begin{array}{r}
\left\langle G\left|\left\langle 0\left|C_{j \uparrow}\left(-2 J_{0} \sigma_{l} \cdot \mathbf{S}_{l}\right) C_{i \uparrow}^{+}\right| 0\right\rangle\right| G\right\rangle=\frac{J_{0}}{2} \delta_{j, l} \delta_{i, l} \\
\left\langle G\left|\left\langle 0\left|C_{j \uparrow}\left(-2 J_{1} \mathbf{S}_{l} \cdot \mathbf{S}_{l+1}\right) C_{i \uparrow}^{+}\right| 0\right\rangle\right| G\right\rangle=-\frac{J_{1}}{2} \delta_{i, j} .
\end{array}
$$

We therefore solve the eigenvalue problem: $H\left|\eta_{j}\right\rangle=$ $E_{j}\left|\eta_{j}\right\rangle$, where $j=1,2, \cdots N_{0}\left(N_{s}+1\right)$. Then, we represent the time dependent total state using eigenvalues and eigenstates as follows: $|\psi(t)\rangle=\sum_{j}\left|\eta_{j}\right\rangle\left\langle\eta_{j} \mid \psi(0)\right\rangle e^{-i E_{j} t}$. Alternatively, the total state at $t$ can be expressed as

$|\psi(t)\rangle=\sum_{i=1}^{N_{0}} \psi_{i}(t) C_{i \uparrow}^{+}|0\rangle|G\rangle+\sum_{l=1}^{N_{s}} \sum_{i=1}^{N_{0}} \psi_{l, i}(t) C_{i \downarrow}^{+}|0\rangle S_{l+}|G\rangle$.

Using this expression we can investigate the dynamics of a particular spin or the sum of all spins in the chain. For example,

$$
\left\langle\psi(t)\left|S_{l z}\right| \psi(t)\right\rangle=-\frac{1}{2} \sum_{i^{\prime}}\left|\psi_{i^{\prime}}\right|^{2}+\frac{1}{2} \sum_{l^{\prime}, i^{\prime}}\left|\psi_{l^{\prime}, i^{\prime}}\right|^{2}\left(2 \delta_{l, l^{\prime}}-1\right),
$$

and the total local spin is $\sum_{l}\left\langle\mathbf{S}_{l}(t)\right\rangle$. Since at $t=0$, $\psi_{l, i}=0$ and $\sum_{i}\left|\psi_{i}\right|^{2}=1,\left\langle S_{l z}(0)\right\rangle=-1 / 2$ is assumed. Thus, $\left\langle S_{z}(0)\right\rangle=\sum_{l}\left\langle S_{l z}(0)\right\rangle=-N_{s} / 2$ and $\left\langle J_{z}(0)\right\rangle=$ $\left(1-N_{s}\right) / 2$.

By way of an example, consider a chain of three spins, ferromagnetically coupled with strength $J_{1}$. As an electron impinges on the three spin system, they interact 
Fig. 6 (Kim et al)

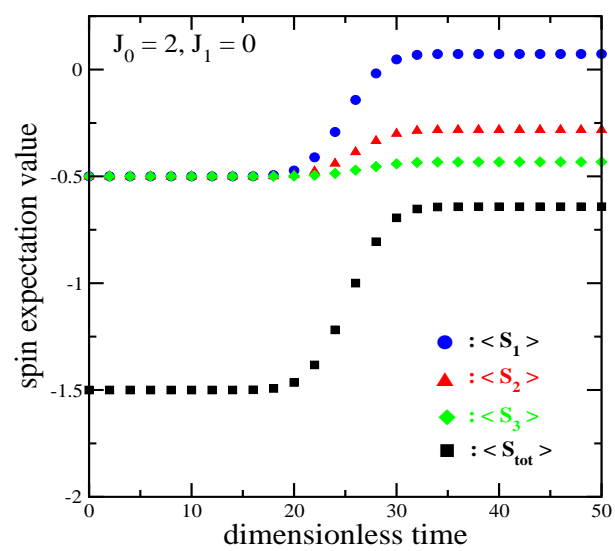

FIG. 6: The spin expectation value $\left\langle S_{z}\right\rangle$ for three spins $S_{i}$ $(i=1,2,3)$ and the total spin $S_{\text {tot }}$. The coupling parameters are $J_{0}=2$ and $J_{1}=0$

Fig. 7 (Kim et al)

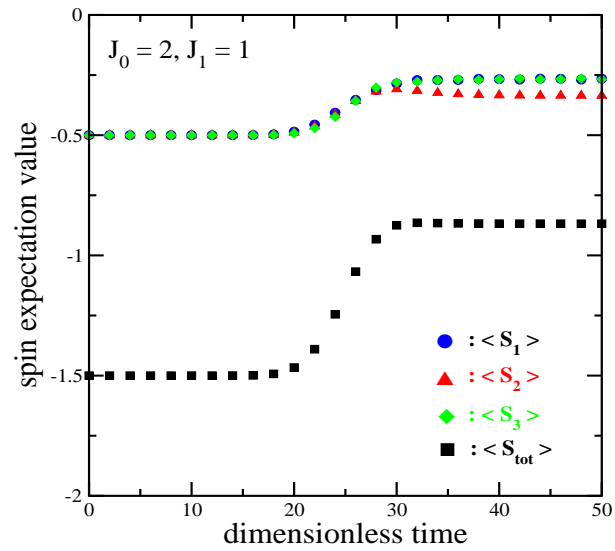

FIG. 7: The spin expectation value $\left\langle S_{z}\right\rangle$ for three spins $S_{i}$ $(i=1,2,3)$ and the total spin $S_{\text {tot }}$. The coupling parameters are $J_{0}=2$ and $J_{1}=1$.

with the electronic spin and change their states. In Fig. 6, we first plot the expectation value of $S_{z}$ for $\mathbf{S}_{i}$ $(i=1,2,3)$, and $\mathbf{S}_{t o t}=\sum_{i=1}^{3} \mathbf{S}_{i}$ for the uncoupled spin case, i.e. with $J_{1}=0$. The electron is coupled to each spin with strength $J_{0}=2$, in units of $t_{0}$. Since there is no coupling between two nearest spins in this instance, each spin evolves independently as a function of time. The spin transfer from the incoming electron to the local spins occurs mostly for $\mathbf{S}_{1}$ while it is minimal for $\mathbf{S}_{3}$.

On the other hand, when the spins are Heisenberg coupled with strength $J_{1}=1$ all three spins participate in the spin-flip scattering with the incoming electron almost to the same degree, as shown in Fig. 7. Interestingly, even though there is an obvious asymmetry (the electron strikes the first spin first) the time evolution of the first $\left(S_{1}\right)$ and the third $\left(S_{3}\right)$ spins are almost identical.

\section{CONCLUSIONS}

In this paper we have described three rather general methods to solve scattering problems on a lattice. The first two are based on single electron-impurity scattering problems, and tell us about the end result of the scattering process. We examined several examples of impurity scattering. In particular, following Giri et al ${ }^{25}$, we explored parameter regions where near resonant transmission is expected to occur for the random trimer model. As is evident from Fig. 3, there are regions in parameter space where near resonant transmission will remain as imperfections arise in the trimer itself. It would be very interesting to examine the width of the resonance for $\mathrm{N}$ such trimers arranged randomly on an infinite lattice, as $\mathrm{N}$ increases.

The third method developed in this paper is numerically intensive, but allows for solution of completely general problems, including those involving spin-flip scattering, as explored briefly in the last section. Moreover, one can monitor the time-dependence of the wave function amplitudes throughout the scattering event. This will be of interest as experimental methods evolve to allow more controlled temporally and spatially resolved measurements. We presented, by way of example the case of a Heisenberg chain of spins, interacting with an itinerant electron (spin current). A natural extension of this calculation could be to include more spins, so as to model an actual magnetized thin film. Generalizing to the case of a spin current is straightforward, so long as we assume the electrons in the current do not interact with one another. This will allow us to address detailed questions about how the spins in the chain reverse their magnetization when subjected to a spin current. These and other questions will be the subject of future investigation.

\section{Acknowledgments}

We wish to thank J. Hirsch for early correspondence concerning the solution of the Schrodinger equation as presented in Ref. 20. W.K. acknowledges A. Mann and M. Revzen for helpful discussions. This work was supported in part by the Natural Sciences and Engineering Research Council of Canada (NSERC), by ICORE (Alberta), and by the Canadian Institute for Advanced Research (CIAR). F.M. is appreciative of the hospitality of the Department of Condensed Matter Physics at the University of Geneva.
1 M. Drescher, M. Hentschel, R. Kienberger, M. Uiberacker, V. Yakovlev, A. Scrinzi, Th. Westerwalbesloh, U.
Kleineberg, U. Heinzmann and F. Krausz, Nature 419, 
$803(2002)$

2 A. Baltuka, Th. Udem, M. Uiberacker, M. Hentschel, E. Goulielmakis, Ch. Gohle, R. Holzwarth, V. S. Yakovlev, A. Scrinzi, T. W. Hansch and F. Krausz, Nature 421, 611 (2003).

3 F.A. Hegmann, Physics in Canada 59, 127 (2003).

${ }^{4}$ M.R. Freeman and W.K. Hiebert, in Spin Dynamics in Confined Magnetic Structures I, edited by B. Hillebrands and K. Ounadjela (Springer, Berlin, 2002).

${ }^{5}$ B.C. Choi, M. Belov, W.K. Hiebert, G.E. Ballentine, and M.R. Freeman, Phys. Rev. Lett. 86, 728 (2001).

${ }^{6}$ See, for example, P.A. Rikvold, G. Brown, S.J. Mitchell, and M.A. Novotny, in Nanostructured Magnetic Materials and their Applications, edited by D. Shi, B. Aktas, L. Pust, and F. Mikailov, Springer Lecture Notes in Physics, Vol. 593 (Springer, Berlin, 2002), p. 164. See also cond-mat/0110103

7 L.-C. Ku and S. A. Trugman, cond-mat/0310226 unpublished.

8 L. Berger, Phys. Rev. B54, 9353, (1996).

9 J.C. Slonczewski, J. Magn. Magn. 159 L1, (1996).

10 J.C. Slonczewski, J. Magn. Magn. 195 L261, (1999).

11 L. Berger, J. Appl. Phys. bf 895521 (2001).

12 J.Z. Sun, Phys. Rev. B 62, 570 (2000).

13 J. Miltat, G. Albuquerque, and A. Thiaville, in Spin Dynamics in Confined Magnetic Structures I, edited by B. Hillebrands and K. Ounadjela (Springer, Berlin, 2002).

14 W. Kim and F. Marsiglio, Europhys. Lett. 69595 (2005).

15 W. Kim and F. Marsiglio, Phys. Rev. B 69212406 (2004).
16 M.D. Stiles and A. Zangwill, Phys. Rev. B 66, 014407 (2002).

17 See, for example, R. Shankar, Principles of Quantum Mechanics, (Plenum Press, New York, 1980).

18 See, for example, G.P. Agrawal, Nonlinear fiber optics (Academic Press, New York 2001). We thank F. Hegmann for pointing this out to us.

19 H.J. Lipkin, Quantum Mechanics: New Approaches to Selected Topics (North-Holland, Amsterdam 1973).

20 J.E. Hirsch, Phys. Rev. B50, 3165 (1994).

21 W. Kim, L. Covaci, and F. Marsiglio, to appear in Phys. Rev. B (cond-mat/0601323).

22 D.H. Dunlap, H.-L. Wu, and P. Phillips, Phys. Rev. Lett. 65, 88 (1990).

23 H.-L. Wu, and P. Phillips, Phys. Rev. Lett. 66, 1366 (1991).

24 H.-L. Wu, W. Goff, and P. Phillips, Phys. Rev. B 45, 1623 (1992).

25 D. Giri, P.K. Datta, and K. Kundu, Phys. Rev. B 48, 14113 (1993).

${ }^{26}$ For an excellent discussion in the continuum limit, see R. Gilmore, Elementary Quantum Mechanics in One Dimension (The Johns Hopkins University Press, Baltimore, 2004).

27 B.L. Burrow and K.W. Sulton, Phys. Rev. B 51, 5732 (1995)

28 Y. Avishai and Y. Tokura, Phys. Rev. Lett. 87, 197203 (2001) 